tapering or other limitations to background treatments have been associated with increased discrimination between treatments and placebo by lowering relative rates of placebo responses. In the current study, CS tapering is required for all patients whose disease has not worsened.

Results Planned enrollment is 360 patients (90 per cohort) at approximately 170 sites in 15 countries. A monitored review of medically appropriate disease activity scoring and tapering of CS is a part of the administration of this trial. Accrual into the PAISLEY study is ongoing.

Conclusions This study will characterize the efficacy and safety of BMS-986165, which works by a novel mechanism of TYK2 inhibition, in patients with moderate to severe manifestations of SLE.

Funding Source(s): Bristol-Myers Squibb

\section{EFFICACY OF CENERIMOD, A SELECTIVE S1P1 RECEPTOR MODULATOR, IN THE MRL/LPR MOUSE MODEL OF SYSTEMIC LUPUS ERYTHEMATOSUS}

Marianne M Martinic*, Sylvie Froidevaux, Estelle Gerossier-Creusat, Enrico Vezzali, Anna Stalder, Ulrich Mentzel. Idorsia Pharmaceuticals Ltd

10.1136/lupus-2019-Ism.6

Background Systemic lupus erythematosus (SLE) is a complex autoimmune disease driven by pathological autoreactive $\mathrm{T}$ and B lymphocytes. Sphingosine 1-phosphate (S1P) regulates the egress of $\mathrm{T}$ and $\mathrm{B}$ lymphocytes from peripheral lymphoid organs. Cenerimod, a potent, selective, and orally active S1P1 receptor modulator that induces receptor internalization, targets this mechanism and prevents lymphocyte exit from lymphoid organs. Based on this rationale, we tested the potential of cenerimod to reduce the pathological manifestations of SLE in the MRL/lpr lupus mouse model.

Methods 7-week-old MRL/lpr mice ( $n=20 /$ group) were assigned to either a vehicle (control) or a cenerimod group and received their respective treatments as food admix ad libitum (around $20-40 \mathrm{mg}$ cenerimod/kg body weight). The study was predefined to end when $20 \%$ mortality or morbidity was reached in one group. Mice were weighed twice a week and blood and urine samples were collected before treatment (week 0), at week 6, and at the end of treatment (week 11). At sacrifice, organs were collected, weighed, and evaluated by flow cytometry or histology. All in vivo readouts and histological data analyses were performed in a blinded fashion.

Results More than 20\% mortality was observed in the control group after 10 weeks of treatment, whereas all mice in the cenerimod group remained alive during this period. Cenerimod-treated mice had significantly fewer circulating immune cells and immune cell infiltrates in the kidney and brain. This translated in preserved organ function as demonstrated by the significantly reduced urine albumin concentrations and kidney and brain histopathological scores. Furthermore, anti-dsDNA antibody, and both plasma BAFF and IFN- levels, two proteins currently targeted clinically, were significantly lower in the cenerimod group.

Conclusions Cenerimod treatment led to increased survival and significantly improved organ pathology in MRL/lpr mice by targeting the S1P1 axis. These results support clinical evaluation of cenerimod for the treatment of SLE.

Funding Source(s): Idorsia Pharmaceuticals Ltd.

\section{MODIFIED IMMUNE CELL THERAPY AMELIORATES MURINE LUPUS NEPHRITIS AND INDUCES REGULATORY CELL SUBSETS}

${ }^{1}$ Claudius Speer*, ${ }^{1}$ Daniela Kim, ${ }^{2}$ Christian Kleist, ${ }^{3}$ Christian Morath, ${ }^{4}$ Anita Schmitt, ${ }^{5}$ Michael Schmitt, ${ }^{1}$ Claudia Sommerer, ${ }^{6}$ Andrea Steinborn, ${ }^{1}$ Florian Kälble, ${ }^{1}$ Christian Nusshag, ${ }^{4}$ Lei Wang, ${ }^{4}$ Alexander Kunz, ${ }^{5}$ Hanns-Martin Lorenz, ${ }^{1}$ Martin Zeier, ${ }^{3}$ Matthias Schaier. 'University of Heidelberg, Department of Nephrology; ${ }^{2}$ University of Heidelberg, Department of Nuclear Medicine, Germany; ${ }^{3}$ University of Heidelberg, Department of Nephrology, Tolerogenix X GmbH; ${ }^{4}$ University of Heidelberg, Department of Hematology and Rheumatology, Tolerogenix $\mathrm{GmbH} ;{ }^{5}$ University of Heidelberg, Department of Hematology and Rheumatology; ${ }^{6}$ University of Heidelberg, Department of Obstetrics and Gynecology

\subsection{6/lupus-2019-Ism.7}

Background Modified immune cells (MICs) are mononuclear cells that gain immunosuppressive properties after incubation with the proliferation inhibitor mitomycin C. We showed that syngeneic MIC cell therapy prevented experimental autoimmune encephalitis (EAE) as well as alloreactive rejection in rat heart and hindlimb and pig kidney transplantation. We now aimed to translate these encouraging investigations to prevent murine lupus nephritis and control autoimmunity by MIC therapy.

Methods Splenocytes of syngeneic NZB/W F1 (BWF1) mice were harvested from a donor, incubated with mitomycin $C$ and injected into recipients tail vein after matching for age and disease activity. Group 1 received no MIC therapy, group 2 standard-dose MIC therapy with $1.5 \times 108 / \mathrm{kg}$ BW once and group 3 repeated MIC therapy with $1.5 \times 108 / \mathrm{kg}$ BW at week 1, 2 and 3, respectively. Group 4 was dosed with repeated MIC therapy before disease onset as preemptive treatment approach. Disease activity was monitored by loss of body weight, protein excretion and serum creatinine. Combined primary endpoint was day 40 post-treatment, protein excretion $3 \mathrm{~g} / \mathrm{L}$ for 2 consecutive weeks and $>30 \%$ loss of original body weight. Histopathology with PAS and HE staining was performed to assess degree of lupus nephritis. In the peripheral blood we determined regulatory cell subsets.

Results MIC therapy prevented the progression of fatal lupus nephritis in BMF1 mice. Protein excretion and serum creatinine were lower in standard-dose group and preemptive group compared to control group whereas repeated MIC therapy in active disease failed to inhibit both significantly. Accomplishment of combined primary endpoint was significantly increased in group $1(67 \%)$ compared to group $2(14 \%)$, group $3(14 \%)$ and group $4(0 \%)$, respectively. Renal architecture was preserved throughout different MIC treatment groups with decreased glomerular and tubular damage scores. After MIC therapy, frequencies of CD8 + CD25+FoxP3 + regulatory $\mathrm{T}$ cells and CD19+CD5+CD1 dhigh regulatory $\mathrm{B}$ cells increased significantly, whereas double negative $\mathrm{T}$ cells were markedly reduced throughout all treatment groups.

Conclusions MIC therapy inhibits progression of active lupus nephritis. Interestingly, preemptive MIC therapy was even able to prevent onset of disease since no significant disease activity was observed at completion of the study. In accordance with our previous pre-clinical EAE experiments and a first inhuman clinical trial in living-donor kidney transplantation (TOL-1 study), MIC therapy enables an in-vivo induction of regulatory cell subsets. This clinically applicable cell therapeutic approach may control lupus nephritis by specifically silencing deleterious autoimmune response.

Funding Source(s): Research grant from TolerogenixX GmbH 